\title{
In Vitro Activity of $\beta$-Lactams in Combination with $\beta$-Lactamase Inhibitors against Mycobacterium tuberculosis Clinical Isolates
}

\author{
Fu Li $\left(\mathbb{D},{ }^{1}\right.$ Li Wan, ${ }^{2}$ Tongyang Xiao $\left(\mathbb{D},{ }^{1}\right.$ Haican Liu $\left(\mathbb{D},{ }^{1}\right.$ Yi Jiang, ${ }^{1}$ \\ Xiuqin Zhao $\mathbb{D}^{1},{ }^{1}$ Ruibai Wang $\left(\mathbb{D},{ }^{1}\right.$ and Kanglin Wan $\mathbb{D}^{1}$ \\ ${ }^{1}$ State Key Laboratory for Infectious Diseases Prevention and Control, Collaborative Innovation Center for Diagnosis and \\ Treatment of Infectious Diseases, National Institute for Communicable Disease Control and Prevention, Chinese Center for \\ Disease Control and Prevention, Beijing 102206, China \\ ${ }^{2}$ Department of Physiology, Xiangya School of Medicine, Central South University, Changsha, Hunan 410078, China
}

Correspondence should be addressed to Ruibai Wang; wangruibai@icdc.cn and Kanglin Wan; wankanglin@icdc.cn

Received 19 February 2018; Accepted 4 June 2018; Published 2 July 2018

Academic Editor: Isabel Portugal

Copyright (C) $2018 \mathrm{Fu} \mathrm{Li}$ et al. This is an open access article distributed under the Creative Commons Attribution License, which permits unrestricted use, distribution, and reproduction in any medium, provided the original work is properly cited.

\begin{abstract}
Objectives. Evaluating the activity of nineteen $\beta$-lactams in combination with different $\beta$-lactamase inhibitors to determine the most potent combination against Mycobacterium tuberculosis (MTB) in vitro. Methods. Drug activity was examined by drug susceptibility test with 122 clinical isolates from China. Mutations of $b l a C$ and drug targets $l d t_{M t 1}, l d t_{M t 2}$, $d a c B 2$, and $c r f A$ were analyzed by nucleotide sequencing. Results. Tebipenem (TBM) in combination with clavulanate (CLA) exhibited the highest anti-TB activity. The MIC of $\beta$-lactam antibiotics was reduced most evidently in the presence of CLA, compared to avibactam (AVI) and sulbactam (SUB). Eight polymorphism sites were identified in blaC, which were not associated with $\beta$-lactams resistance. Interestingly, one strain carrying G514A mutation in blaC was highly susceptible to $\beta$-lactams regardless of the presence of inhibitors. The transpeptidase encoding genes, $l d t_{M t 1}, l d t_{M t 2}$, and $d a c B 2$, harboured three mutations, two mutations, and one mutation, respectively, but no correlation was found between these mutations and drug resistance. Conclusion. The activity of $\beta$-lactams against MTB and different synergetic effect of $\beta$-lactamase inhibitors were indicated. TBM/CLA exhibited the most activity and has a great prospect in developing novel anti-TB regimen; however, further clinical research is warranted. Moreover, the resistance to the $\beta$-lactam antibiotics might not be conferred by single target mutation in MTB and requires further studies.
\end{abstract}

\section{Introduction}

Tuberculosis (TB) is a great threat to the global public health that caused an estimated 1.3 million deaths in 2016 [1]. Drug resistance has been a major obstacle in the progress of eliminating $\mathrm{TB}$, especially due to the emergence of multidrugresistant (MDR) and extensively drug-resistant (XDR) MTB worldwide [2-4]. With the rising incidence of drug-resistant $\mathrm{TB}$, the traditional first-line and second-line anti-TB drugs fail to fulfill the current needs of TB treatments. In such cases, developing novel drugs or drug regimens is urgent and necessary for effective control of TB. Although significant progress has been made in identification of several new anti-TB compounds, such as bedaquiline, delamanid, and pretomanid, which are in phase II or III trials $[5,6]$, long duration and high expenses lead to slow development of new drugs. Repurposing the currently used antibiotics may contribute to the development of novel anti-TB regimens.

$\beta$-lactams disrupt the cell wall biosynthesis of bacteria by preventing the formation of peptide cross-links between the adjacent polysaccharide chains in the peptidoglycan layer [7]. In Mycobacterium tuberculosis (MTB), two kinds of transpeptidase, the L,D-transpeptidase (mainly encoded by $l d t_{M t 1}$ and $l d t_{M t 2}$ ) and the D,D-transpeptidase (encoded by dacB2), catalyze the cell wall biosynthesis $[8,9]$. The L,Dtranspeptidase, which is responsible for the formation of over $80 \%$ transpeptide linkages ( $3 \longrightarrow 3$ linkages) in MTB, is the target of carbapenems [10-12]. The D,D-transpeptidase that 
mainly catalyzes the formation of $4 \longrightarrow 3$ linkages is a kind of penicillin-binding proteins (PBPs), which can be inhibited by meropenem (MEM) [13].

The resistance to $\beta$-lactam antibiotics encompasses the activity of $\beta$-lactamases. Thousands of different $\beta$ lactamases have been identified in various environmental and pathogenic species of bacteria [14]. MTB is intrinsically resistant to $\beta$-lactams due to the expression of endogenous $\beta$-lactamase $\mathrm{BlaC}$ active against a broad spectrum of $\beta$ lactams. CLA, AVI, and SUB are $\beta$-lactamase inhibitors that inhibit the hydrolysis of $\beta$-lactams. Recent studies have reported that a combination of the $\beta$-lactams and $\beta$ lactamase inhibitors is effective against MTB in vitro and in vivo [15-17]. The efficacy, safety, and tolerability of carbapenems, such as MEM, imipenem, and ertapenem (ETP), against MTB have been evaluated in several clinical studies [18]. Amoxicillin/clavulanate (AMX/CLA) combination has been evaluated to be efficient against MTB in vitro and is thus categorized into Group 5 anti-TB drugs by WHO [19, $20]$. This suggests that combinations of $\beta$-lactams and $\beta$ lactamase inhibitors are prospective candidates in developing more effective treatment against TB.

In this study, to determine the most active and applicable combination of $\beta$-lactam and $\beta$-lactamase inhibitors, the in vitro anti-TB activity of nineteen $\beta$-lactams belonging to different subclasses either alone or in combination with different $\beta$-lactamase inhibitors was tested using drug susceptibility tests. A total of $122 \mathrm{MTB}$ clinical isolates collected from China were used. Mutations in blaC and three main $\beta$-lactams targets, $l d t_{M t 1}, l d t_{M t 2}$, and $d a c B 2$, were analyzed to determine the relationship between drug target mutations and $\beta$-lactam resistance. In addition, T184A mutation in an unannotated gene, $\operatorname{crf} A$, reported to confer carbapenem resistance in MTB was also detected.

\section{Materials and Methods}

2.1. Strains. A total of 122 identified clinical MTB isolates, including 47 susceptible (S), $64 \mathrm{MDR}$, and $11 \mathrm{XDR}$ isolates, used in this study were collected from seven provincial TB hospitals of China in Beijing, Anhui, Fujian, Guizhou, Henan, Xinjiang, and Sichuan. All the strains were preserved in the strain bank of National Institute for Communicable Disease Control and Prevention, Chinese Center for Disease Control and Prevention.

2.2. $\beta$-Lactams and $\beta$-Lactamase Inhibitors. MEM was purchased from Sigma-Aldrich Co. (St Louis, MO, USA). Tebipenem, doripenem, biapenem, ertapenem, amoxicillin, ampicillin, methicillin, oxacillin, cloxacillin, nafcillin, dicloxacillin, piperacillin, cephalexin, cefuroxime, cefixime, cefpirome, aztreonam, and moxalactam and three $\beta$-lactamase inhibitors, CLA, AVI, and SUB, used in this study were purchased from MCE Co. (Monmouth Junction, NJ, USA) (Table 1).

2.3. Drug Susceptibility Test. Drug susceptibility test was performed by broth microdilution method using 96-well microplate. Briefly, a loop of MTB culture in the late log phase
TABLE 1: The $\beta$-lactams and $\beta$-lactamase inhibitors for drug susceptibility tests.

\begin{tabular}{lcc}
\hline Classification & Agent & Generation \\
\hline Carbapenem & Tebipenem & \\
& Meropenem & \\
Doripenem & \\
& Biapenem & \\
Ertapenem & \\
Penicillin & Amoxicillin & \\
& Ampicillin & \\
& Methicillin & \\
& Oxacillin & \\
& Cloxacillin & \\
& Nafcillin & \\
& Dicloxacillin & \\
Cephalosporin & Piperacillin & \\
& Cephalexin & First generation \\
& Cefuroxime & Second generation \\
& Cefixime & Third generation \\
& Cefpirome & Fourth generation \\
Oxactamase inhibitor & Aztreonam & \\
& Moxalactam & \\
& Clavulanate & \\
& Avibactam & \\
& Sulbactam & \\
& & \\
& &
\end{tabular}

was fully ground and adjusted with saline to a cell density of $3 \times 10^{8}$ cells $/ \mathrm{mL}$ ( $1 \mathrm{McF}$ arland standard). The suspension was diluted 1:20 with 7H9 broth (Difco, Detroit, MI, USA) containing 10\% OADC (BD, Franklin Lakes, NJ, USA). Six groups of serial twofold drug dilutions were prepared in the $7 \mathrm{H} 9$ broth containing either five different constant concentrations of each $\beta$-lactamase inhibitor or no inhibitor at all. The final reaction mix contained $100 \mu \mathrm{l}$ of drug solution and equal volume of bacterial suspension in each well. The plates were sealed and incubated at $37^{\circ} \mathrm{C}$ for 7 days. Then the indicator $(20 \mu \mathrm{l}$ Alamar Blue mixed with $50 \mu \mathrm{l} 5 \%$ Tween80 ) was added to the drug-containing group when the drugfree control showed color change (blue to pink). The lowest drug concentration that inhibited the strain growth and prevented color change was recorded as the MIC value. Drug susceptibility tests for each strain were repeated twice.

The final concentrations of tebipenem ranged from 0.125 to $16 \mu \mathrm{g} / \mathrm{ml}$, and for the other carbapenems, 0.25 to 32 $\mu \mathrm{g} / \mathrm{ml}$ concentrations were tested in drug susceptibility tests. Ampicillin (AMP) and cefuroxime (CXM) were used at concentrations ranging from 0.5 to $64 \mu \mathrm{g} / \mathrm{ml}$ and the other chemicals were tested at concentrations ranging from 1 to 128 $\mu \mathrm{g} / \mathrm{ml}$. The final concentration of each $\beta$-lactamase inhibitor was $0.625 \mu \mathrm{g} / \mathrm{ml}, 1.25 \mu \mathrm{g} / \mathrm{ml}, 2.5 \mu \mathrm{g} / \mathrm{ml}, 5 \mu \mathrm{g} / \mathrm{ml}$, and $10 \mu \mathrm{g} / \mathrm{ml}$, respectively.

2.4. Polymerase Chain Reaction (PCR) and Sequencing. Genomic DNA was isolated from mycobacterial cultures by boiling method and was used for amplification of blaC, 
TABle 2: Primers and conditions used for amplification and sequencing.

\begin{tabular}{|c|c|c|c|c|c|}
\hline \multirow{2}{*}{ Primers } & \multirow{2}{*}{ Sequence $\left(5^{\prime}-3^{\prime}\right)$} & \multicolumn{3}{|c|}{ PCR conditions } & \multirow{2}{*}{ Product length (bp) } \\
\hline & & Denaturation $^{\mathrm{a}}(\mathrm{s})$ & Annealing $\left({ }^{\circ} \mathrm{C}, \mathrm{s}\right)$ & Elongation $^{\mathrm{b}}(\mathrm{s})$ & \\
\hline $\mathrm{BlaC}-\mathrm{F}$ & ATGCGCAACAGAGGATTCGGTC & \multirow{2}{*}{30} & \multirow{2}{*}{61,30} & \multirow{2}{*}{65} & \multirow{2}{*}{924} \\
\hline BlaC-R & CTATGCAAGCACACCGGCAACG & & & & \\
\hline $\mathrm{Ldt}_{\mathrm{Mt1}}-\mathrm{F}$ & ATGCGTCGAGTGGTTCGTTATC & \multirow{2}{*}{30} & \multirow{2}{*}{58,30} & \multirow{2}{*}{55} & \multirow{2}{*}{756} \\
\hline $\mathrm{Ldt}_{\mathrm{Mt1}}-\mathrm{R}$ & CTAGCCGACCACCTCAATGG & & & & \\
\hline $\mathrm{Ldt}_{\mathrm{Mt2} 2}-\mathrm{F}$ & ATGCCAAAGGTGGGGATTGC & \multirow[t]{2}{*}{30} & \multirow{2}{*}{59,30} & \multirow[t]{2}{*}{90} & \multirow{2}{*}{1227} \\
\hline $\mathrm{Ldt}_{\mathrm{Mt2}}-\mathrm{R}$ & TTACGCCTTGGCGTTACCGGC & & & & \\
\hline DacB2-F & ACCAGCAACTGCTGGATTTC & \multirow{2}{*}{30} & \multirow{2}{*}{60,30} & \multirow{2}{*}{85} & \multirow{2}{*}{1196} \\
\hline DacB2-R & CGTTGATGACCAACGTCTTC & & & & \\
\hline CrfA-F & ACCCGGCTCACAGAGAATCG & \multirow[t]{2}{*}{30} & \multirow{2}{*}{60,30} & \multirow{2}{*}{40} & \multirow{2}{*}{457} \\
\hline CrfA-R & TATCACCGGTAGGCCATGC & & & & \\
\hline
\end{tabular}

Note: ${ }^{a}$ : denaturation temperature was $94^{\circ} \mathrm{C} ;{ }^{b}$ : elongation temperature was $72^{\circ} \mathrm{C}$. All PCRs were performed for 31 cycles and each reaction was initialized by denaturation at $94^{\circ} \mathrm{C}$ for $10 \mathrm{~min}$ and terminated with a final elongation step at $72^{\circ} \mathrm{C}$ for $10 \mathrm{~min}$.

$l d t_{M t 1}, l d t_{M t 2}, d a c B 2$, and $c r f A$ by polymerase chain reaction (PCR). The primer sets and conditions for amplification are shown in Table 2. The PCR products were sequenced (TSINGKE Biological Technology, Beijing, China) and the gene polymorphisms were identified by aligning with the reference strain H37Rv (GenBank accession no. NC_000962) using MEGA 7.0 software.

\section{Results}

3.1. Determination of Inhibitors' Concentrations. The effective concentration of each $\beta$-lactamase inhibitor was determined by drug susceptibility tests against six MTB clinical isolates with nineteen $\beta$-lactams either alone or in combination with five different concentrations of $\beta$-lactamase inhibitors. The results showed that the MICs did not change when $5 \mu \mathrm{g} / \mathrm{ml}$ concentration of inhibitors was used (Table 3 ). Thus, we decided to use inhibitors at a fixed final concentration of $5 \mu \mathrm{g} / \mathrm{ml}$ in the subsequent experiments. All the carbapenems, AMX, ampicillin (AMP), and cefuroxime (CXM) were selected to perform further tests, because the other $\beta$-lactams showed no activity against MTB in the initial screening.

3.2. MIC Profiles of $\beta$-Lactams against Clinical Isolates. The MIC profiles of the clinical isolates are shown in Table 4. In the case of carbapenems, TBM and biapenem (BIA) showed better activity with $\mathrm{MIC}_{90}$ of $16 \mu \mathrm{g} / \mathrm{ml}$ when they were used alone. The MICs of carbapenems were greatly reduced (48 folds), except for ETP (1-4 folds), in the presence of $\beta$ lactamase inhibitors. TBM in combination with CLA showed the most inhibitory activity with $\mathrm{MIC}_{50}$ and $\mathrm{MIC}_{90}$ of $1 \mu \mathrm{g} / \mathrm{ml}$ and $2 \mu \mathrm{g} / \mathrm{ml}$, respectively, followed by BIA $(2 \mu \mathrm{g} / \mathrm{ml}$ and 4 $\mu \mathrm{g} / \mathrm{ml})$ and doripenem (DOR) $(2 \mu \mathrm{g} / \mathrm{ml}$ and $8 \mu \mathrm{g} / \mathrm{ml})$. MEM in combination with CLA also exhibited good activity with $\mathrm{MIC}_{50}$ and $\mathrm{MIC}_{90}$ of $2 \mu \mathrm{g} / \mathrm{ml}$ and $8 \mu \mathrm{g} / \mathrm{ml}$, respectively, but it was lower than that of TBM and BIA. ETP in combination with any of the inhibitors showed the least activity with $\mathrm{MIC}_{90}$ of $>16 \mu \mathrm{g} / \mathrm{ml}$. In the case of penicillins and cephalosporins, AMX/CLA showed highest activity against MTB with MIC $_{50}$ and $\mathrm{MIC}_{90}$ of $2 \mu \mathrm{g} / \mathrm{ml}$ and $8 \mu \mathrm{g} / \mathrm{ml}$, respectively, followed by AMP/CLA $(4 \mu \mathrm{g} / \mathrm{ml}$ and $16 \mu \mathrm{g} / \mathrm{ml})$. CXM alone or in combination with any of the inhibitors showed no anti-TB activity even when the highest drug concentration was tested.

Comparison of the synergistic effect of the three different $\beta$-lactamase inhibitors shows that CLA exhibited the most potent activity in inhibiting the $\beta$-lactamase. Overall, the MICs of $\beta$-lactams were reduced most prominently (4-32 folds) in the presence of CLA, followed by AVI (4-8 folds). However, the least activity was observed in case of SUB as $\mathrm{MIC}_{50}$ and $\mathrm{MIC}_{90}$ of all the tested drug combinations with SUB showed lesser reduction (2-8 folds) than that in the presence of CLA and AVI, especially when used in combination with TBM, BIA, and AMP.

3.3. Relationship between Drug Target Mutation and Resistance. Since the $\beta$-lactamase $\mathrm{BlaC}, \mathrm{L}, \mathrm{D}$-transpeptidase, and $\mathrm{D}, \mathrm{D}$-transpeptidase are potential targets of $\beta$-lactams, the encoding genes, including $b l a C, l d t_{M t 1}, l d t_{M t 2}$, and $d a c B 2$, were analyzed to illustrate the role of drug target mutations in the emergence of the $\beta$-lactams resistance in MTB. In addition, mutation at position T184A in an unannotated protein encoding gene, $\operatorname{crf} A$, which was earlier reported to confer carbapenem resistance in MTB [21], was also analyzed. Mutations in the five genes are shown in Table 5 and the MIC profiles of the mutant strains are listed in the supplementary materials. As observed, 16.4\% (20/122) of clinical isolates carried 8 mutation sites in blaC. The silent mutation, C786T (11 isolates), was the most predominant mutation. Three nonsynonymous mutations, T333G, T492A, and G514A, were observed in blaC, while none of these mutations was related to $\beta$-lactam resistance. Interestingly, one strain, GZ10116, which carried G514A mutation, was highly susceptible to $\beta$ lactams irrespective of the presence of inhibitors.

There were 1, 1, and 2 strains harbouring A111G, G448A, and C659T mutations, respectively, on $l d t_{M t t} .1$ strain and 4 strains, respectively, harboured C594T and A776G mutation in $l d t_{M t 2}$. Only one strain, which harboured A776G mutation in $l d t_{M t 2}$, was resistant to all the combinations of $\beta$-lactams and inhibitors. However, we did not find any mutation associated with the $\beta$-lactam resistance in $l d t_{M t 1}$ and $l d t_{M t 2}$. 


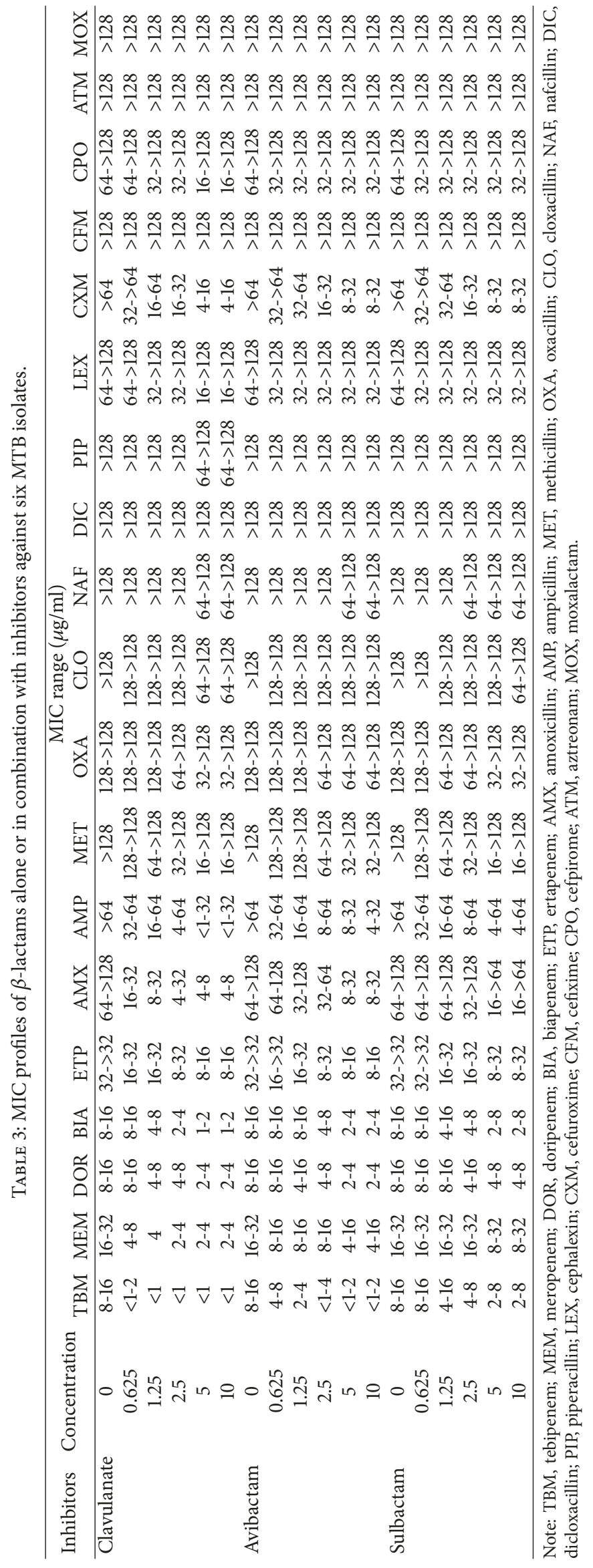


TABLE 4: MICs of $\beta$-lactams alone or in combination with a fixed concentration of $5 \mu \mathrm{g} / \mathrm{ml}$ of the inhibitor against $122 \mathrm{MTB}$ isolates.

\begin{tabular}{|c|c|c|c|c|}
\hline Antibiotic & Inhibitor & $\begin{array}{c}\text { MIC range } \\
(\mu \mathrm{g} / \mathrm{ml})\end{array}$ & $\begin{array}{c}\mathrm{MIC}_{50} \\
(\mu \mathrm{g} / \mathrm{ml})\end{array}$ & $\begin{array}{c}\mathrm{MIC}_{90} \\
(\mu \mathrm{g} / \mathrm{ml})\end{array}$ \\
\hline \multirow[t]{4}{*}{ TBM } & None & $0.5->16$ & 8 & 16 \\
\hline & CLA & $0.125->16$ & 1 & 2 \\
\hline & AVI & $0.125->16$ & 1 & 2 \\
\hline & SUB & $0.5->16$ & 2 & 4 \\
\hline \multirow[t]{4}{*}{ BIA } & None & $2->32$ & 8 & 16 \\
\hline & CLA & $0.25->32$ & 2 & 4 \\
\hline & AVI & $0.5->32$ & 2 & 4 \\
\hline & SUB & $0.5->32$ & 4 & 8 \\
\hline \multirow[t]{4}{*}{ DOR } & None & $2->32$ & 16 & 16 \\
\hline & CLA & $0.5->32$ & 2 & 8 \\
\hline & AVI & $0.5->32$ & 4 & 8 \\
\hline & SUB & $0.5->32$ & 4 & 16 \\
\hline \multirow[t]{4}{*}{ MEM } & None & $4->32$ & $>32$ & $>32$ \\
\hline & CLA & $0.25->32$ & 2 & 8 \\
\hline & AVI & $1->32$ & 4 & 16 \\
\hline & SUB & $4->32$ & 8 & 16 \\
\hline \multirow[t]{4}{*}{ ETP } & None & $8->32$ & $>32$ & $>32$ \\
\hline & CLA & $2->32$ & 8 & 16 \\
\hline & AVI & $4->32$ & 8 & 16 \\
\hline & SUB & $4->32$ & 16 & 32 \\
\hline \multirow[t]{4}{*}{ AMX } & None & $8->64$ & $>64$ & $>64$ \\
\hline & CLA & $<0.5-32$ & 2 & 8 \\
\hline & AVI & $<0.5-64$ & 8 & 16 \\
\hline & SUB & $2->64$ & 8 & 32 \\
\hline \multirow[t]{4}{*}{ AMP } & None & $32->64$ & 64 & $>64$ \\
\hline & CLA & $0.5->64$ & 4 & 16 \\
\hline & AVI & $0.5->64$ & 8 & 16 \\
\hline & SUB & $0.5->64$ & 16 & 32 \\
\hline \multirow[t]{4}{*}{ CXM } & None & $16->64$ & $>64$ & $>64$ \\
\hline & CLA & $1->64$ & 32 & $>64$ \\
\hline & AVI & $2->64$ & 32 & $>64$ \\
\hline & SUB & $4->64$ & 64 & $>64$ \\
\hline
\end{tabular}

Note: $\mathrm{MIC}_{50 / 90}$, MICs that inhibit $50 \%$ and $90 \%$ of the isolates, respectively.

Only one mutation, T659A, was found on $d a c B 2$ in four strains, but it was also not associated with the $\beta$-lactam resistance. In addition, the results showed that no mutation was detected in crfA among all the strains in our study. We also did not find any strain harbouring mutations in the blaC and transpeptidase genes simultaneously.

\section{Discussion}

Novel drugs or regimens are urgently needed due to the rise in the prevalence of multidrug-resistant TB worldwide. $\beta$ lactams are widely used and well-tolerated antibiotics used for controlling clinical Gram-positive and Gram-negative
TABLE 5: Mutations on blaC, $l d t_{M t 1}, l d t_{M t 2}$, and $d a c B 2$ genes.

\begin{tabular}{|c|c|c|c|}
\hline Gene & Substitution & Amino acid change & Number of mutants \\
\hline \multirow[t]{8}{*}{ blaC } & G183A & Leu61Leu & 1 \\
\hline & T333G & Ser111Arg & 3 \\
\hline & $\mathrm{C} 354 \mathrm{~T}$ & Ser118Ser & 1 \\
\hline & G486C & Ala162Ala & 1 \\
\hline & T492A & Phe164Leu & 1 \\
\hline & G514A & Gly172Ser & 1 \\
\hline & C610T & Leu204Leu & 1 \\
\hline & C786T & Ile262Ile & 11 \\
\hline \multirow[t]{3}{*}{$l d t_{M t 1}$} & A111G & Pro37Pro & 1 \\
\hline & G448A & Ala150Thr & 1 \\
\hline & C659T & Ala220Val & 2 \\
\hline \multirow[t]{2}{*}{$l d t_{M t 2}$} & C594T & Gly198Gly & 1 \\
\hline & A776G & Asp259Gly & 4 \\
\hline dacB2 & T659A & Leu220Gln & 4 \\
\hline
\end{tabular}

bacterial infections [22]. Recently, the combination of $\beta$ lactamase inhibitor and $\beta$-lactam was shown to be effective in restoring the potency of $\beta$-lactam against MTB [1517]. In the present study, nineteen commercially available $\beta$-lactams and three $\beta$-lactamase inhibitors were screened in vitro to identify the most potent combination against MTB. The results show that carbapenems, except for ETP, exhibited excellent activity against MTB clinical strains. This may be due to the inactivation of multiple enzymes by carbapenems, whereas the other $\beta$-lactams did not inhibit the L,D-transpeptidase in MTB [10]. The activity of AMX was also improved notably in the presence of clavulanate. While the efficacy of AMX/CLA against clinical TB remains controversial, carbapenems may serve as an optimal alternative in developing novel anti-TB regimens. The results showed that all the tested cephalosporins in our study were inactive against MTB. Dincer et al. [23] reported that cefazolin in combination with CLA was active against H37Ra. This may be due to the different strain tested and different methodology used. Additionally, the concentration of CLA, that is, 32 $\mu \mathrm{g} / \mathrm{ml}$, was much higher than the concentration $(5 \mu \mathrm{g} / \mathrm{ml})$ used in our test. This suggests that a more universal drug susceptibility testing protocol for determining the MIC of $\beta$ lactams against MTB is needed in the future.

Carbapenems displayed a variable activity in this study. TBM in combination with CLA exhibited the highest activity against MTB, followed by BIA and DOR. MIC $_{90}$ of TBM/CLA was $2 \mu \mathrm{g} / \mathrm{ml}$, which can be easily obtained in plasma with the administration of a single dose of $200 \mathrm{mg}$ TBM pivoxil granules [24]. Comparable activity to that of TBM/CLA was observed in case of BIA/CLA. The maximum plasma concentration $\left(C_{\max }\right)$ of BIA after a single intravenous dose was $17.4 \mu \mathrm{g} / \mathrm{ml}$ [25], which exceeded $\mathrm{MIC}_{90}(2 \mu \mathrm{g} / \mathrm{ml})$ of BIA/CLA. Both of BIA and TBM are stable against renal dehydropeptidase-I and are also safe for central nervous system, since they do not exhibit competitive binding to the $\gamma$-aminobutyric acid (GABA) receptors [26]. However, BIA requires intravenous administration that is not easily complied with by the patients. MEM/CLA was previously 
reported to be effective against MDR-TB. The $C_{\max }$ and AUC values of MEM were $26 \mu \mathrm{g} / \mathrm{ml}$ and 27.2-32.4 $\mu \mathrm{g} \cdot \mathrm{h} / \mathrm{ml}$, respectively, after being administered by an intravenous infusion of $500 \mathrm{mg}$ of MEM [27]. In our results also, MEM/CLA exhibited potent activity $\left(\mathrm{MIC}_{90}, 8 \mu \mathrm{g} / \mathrm{ml}\right)$ against $\mathrm{MTB}$ but it was weaker than that of TBM/CLA, which is consistent with previous study [28]. This may be explained by lower $K_{\mathrm{m}}$ and less breakdown of the covalent tebipenem-BlaC adduct, in contrast to that of MEM, thus suggesting a higher activity of TBM against MTB $[17,29]$. Oral administration of the TBM pivoxil had a better protective effect than meropenem in all the tried sepsis models, due to its greater tissue distribution [30]. Hence, as an oral carbapenem, TBM may be a better option in developing new anti-TB regimen.

MTB harbours a chromosomally encoded $\beta$-lactamase, which is selectively susceptible to $\beta$-lactamase inhibitors. In this study, varied synergistic effects of three different $\beta$ lactamase inhibitors were observed. Consistent with previous study [28], the greatest reduction in MIC was achieved by CLA in combination with $\beta$-lactams, especially in AMX/CLA (32-fold in $\mathrm{MIC}_{50}$ ). AVI, a newly developed $\beta$-lactamase inhibitor, combining with ceftazidime exhibited good activity against Enterobacteriaceae and Pseudomonas aeruginosa [31]. However, a combination of AVI with $\beta$-lactams displayed a lesser reduction in MIC of $\beta$-lactams compared to CLA. A recent report has demonstrated that the efficiency of AVI was lower than that of CLA due to its bulky rings that may influence its binding to BlaC [32]. The unstable covalent adduct of SUB and BlaC may also explain its lesser inhibiting activity than that of CLA [33]. Thus, CLA seems to be the most suitable in developing anti-TB regimens encompassing $\beta$-lactams. In addition, the concentration of $5 \mu \mathrm{g} / \mathrm{ml}$ of CLA used in the current study was lower than the $C_{\max }$ of $5.9 \mu \mathrm{g} / \mathrm{ml}$ achieved after a $250 \mathrm{mg}$ three times a day dosage, suggesting that it can be easily achieved in clinical use [34].

For investigating the relationship between mutations in the $\beta$-lactam targets and the drug susceptibility of MTB clinical isolates, $b l a C, l d t_{M t 1}, l d t_{M t 2}$, and $d a c B 2$ in $122 \mathrm{MTB}$ clinical isolates were sequenced and analyzed. BlaC hydrolyzes the $\beta$-lactam ring, which is essential for the activity of $\beta$-lactam antibiotics, resulting in the $\beta$-lactams resistance in MTB. Eight different mutations were observed in blaC. However, we did not find any association between these mutations and $\beta$ lactam resistance. Interestingly, one of the strains harbouring G514A (Gly172Ser) mutation was highly susceptible to $\beta$ lactam antibiotics in either the presence or the absence of the inhibitors. Zhang et al. [35] have reported that the S111R mutation on BlaC may be associated with an increased susceptibility of MTB. Considering that the mutation Gly172Ser may affect the interaction of BlaC and $\beta$-lactams [36], we may speculate that this amino change leads to a conformation change in BlaC, which influences its activity.

Mutation on target gene often confers drug resistance to bacteria. Four clinical MTB strains were found to carry mutation A776G in $l d t_{M t 2}$ and two of them were susceptible to $\beta$-lactams. Similarly, among the dacB2 mutants, one exhibited resistance, while the other exhibited susceptibility to $\beta$-lactams. However, we did not find any correlation between these mutations and the $\beta$-lactams resistance. In this study, we only tested four potential targets and none of the strains harboured mutations simultaneously on all the four genes. It is reported that the $\beta$-lactam antibiotics have to bind to at least three of the four identified PBPs to be effective [37]. Considering the multiple targets of $\beta$ lactams, we may speculate that the drug resistance may not be conferred by mutation in a single target. Further studies are required to demonstrate the role of drug target mutations in the development of $\beta$-lactam resistance in MTB in order to consider the inclusion of $\beta$-lactams in the anti-TB treatment regimens.

\section{Conclusions}

In summary, varied activities of carbapenems, penicillins, and cephalosporins alone or in combination with $\beta$ lactamase inhibitors against MTB were observed. TBM in combination with CLA showed the most remarkable activity and has a good prospect in developing novel anti-TB regimens. Further studies are warranted to investigate the efficacy of TBM/CLA in the clinical trials. Additionally, resistance to the $\beta$-lactams may not be conferred by single target mutation in MTB and thus requires further investigation.

\section{Data Availability}

The datasets used to support the findings of this study are available upon request from the corresponding author.

\section{Conflicts of Interest}

The authors declare that there are no conflicts of interest regarding the publication of this manuscript.

\section{Acknowledgments}

This work was financially supported by the Key project of the State Key Laboratory for Infectious Disease Prevention and Control (2014SKLID104) and the project of the National Key Programs of Mega Infectious Diseases (2013ZX10003002001).

\section{Supplementary Materials}

The details of mutations and the MIC profile of each mutant in this study are listed in the supplementary material file. (Supplementary Materials)

\section{References}

[1] World Health Organization, Global tuberculosis report, WHO Document, World Health Organization, 2017.

[2] L. Wang, H. Zhang, Y. Ruan et al., "Tuberculosis prevalence in China, 1990-2010; a longitudinal analysis of national survey data," The Lancet, vol. 383, no. 9934, pp. 2057-2064, 2014.

[3] T. Cohen, H. E. Jenkins, C. Lu, M. McLaughlin, K. Floyd, and M. Zignol, "On the spread and control of MDR-TB epidemics: An examination of trends in anti-tuberculosis drug resistance 
surveillance data," Drug Resistance Updates, vol. 17, no. 4-6, pp. 105-123, 2014.

[4] K. Dheda, T. Gumbo, N. R. Gandhi et al., "Global control of tuberculosis: from extensively drug-resistant to untreatable tuberculosis," The Lancet Respiratory Medicine, vol. 2, no. 4, pp. 321-338, 2014.

[5] S. Tiberi, R. Buchanan, J. A. Caminero et al., "The challenge of the new tuberculosis drugs," La Presse Médicale, vol. 46, no. 2, pp. e41-e51, 2017.

[6] A. Zumla, J. Chakaya, R. Centis et al., “Tuberculosis treatment and management-an update on treatment regimens, trials, new drugs, and adjunct therapies," The Lancet Respiratory Medicine, vol. 3, no. 3, pp. 220-234, 2015.

[7] M. I. Page, "The Reactivity of $\beta$-Lactams, the Mechanism of Catalysis and the Inhibition of $\beta$-Lactamases," Current Pharmaceutical Design, vol. 5, no. 11, pp. 895-913, 1999.

[8] J.-L. Mainardi, R. Villet, T. D. Bugg, C. Mayer, and M. Arthur, "Evolution of peptidoglycan biosynthesis under the selective pressure of antibiotics in Gram-positive bacteria," FEMS Microbiology Reviews, vol. 32, no. 2, pp. 386-408, 2008.

[9] M. Lavollay, M. Arthur, M. Fourgeaud et al., "The peptidoglycan of stationary-phase Mycobacterium tuberculosis predominantly contains cross-links generated by L,D-transpeptidation," Journal of Bacteriology, vol. 190, no. 12, pp. 4360-4366, 2008.

[10] W.-J. Li, D.-F. Li, Y.-L. Hu, X.-E. Zhang, L.-J. Bi, and D.-C. Wang, "Crystal structure of L,D-transpeptidase $\mathrm{Ldt}_{\mathrm{Mt} 2}$ in complex with meropenem reveals the mechanism of carbapenem against Mycobacterium tuberculosis," Cell Research, vol. 23, no. 5, pp. 728-731, 2013.

[11] J. F. Fisher and S. Mobashery, "beta-Lactam Resistance Mechanisms: Gram-Positive Bacteria and Mycobacterium tuberculosis," Cold Spring Harbor Perspectives in Medicine, vol. 6, no. 5, 2016.

[12] V. Dubée, S. Triboulet, J.-L. Mainardi et al., "Inactivation of Mycobacterium tuberculosis L,D-transpeptidase Ldt $_{\mathrm{Mtl}}$ by carbapenems and cephalosporins," Antimicrobial Agents and Chemotherapy, vol. 56, no. 8, pp. 4189-4195, 2012.

[13] P. Kumar, K. Arora, J. R. Lloyd et al., "Meropenem inhibits D,D-carboxypeptidase activity in Mycobacterium tuberculosis," Molecular Microbiology, vol. 86, no. 2, pp. 367-381, 2012.

[14] C. Brandt, S. D. Braun, C. Stein et al., "In silico serine $\beta$ lactamases analysis reveals a huge potential resistome in environmental and pathogenic species," Scientific Reports, vol. 7, p. 43232, 2017.

[15] P. Kumar, A. Kaushik, E. P. Lloyd et al., "Non-classical transpeptidases yield insight into new antibacterials," Nature Chemical Biology, vol. 13, no. 1, pp. 54-61, 2017.

[16] A. Kaushik, N. C. Ammerman, R. Tasneen et al., "In vitro and in vivo activity of biapenem against drug-susceptible and rifampicin-resistant Mycobacterium tuberculosis," Journal of Antimicrobial Chemotherapy, vol. 72, no. 8, pp. 2320-2325, 2017.

[17] J.-E. Hugonnet, L. W. Tremblay, H. I. Boshoff, C. E. Barry III, and J. S. Blanchard, "Meropenem-clavulanate is effective against extensively drug-resistant Mycobacterium tuberculosis," Science, vol. 323, no. 5918, pp. 1215-1218, 2009.

[18] G. Sotgiu, L. D’Ambrosio, R. Centis et al., "Carbapenems to treat multidrug and extensively drug-resistant tuberculosis: A systematic review," International Journal of Molecular Sciences, vol. 17, no. 3, article no. 373, 2016.

[19] H. F. Chambers, T. Kocagöz, T. Sipit, J. Turner, and P. C. Hopewell, "Activity of amoxicillin/clavulanate in patients with tuberculosis," Clinical Infectious Diseases, vol. 26, no. 4, pp. 874879, 1998.

[20] P. R. Donald, F. A. Sirgel, A. Venter et al., "Early bactericidal activity of amoxicillin in combination with clavulanic acid in patients with sputum smear-positive pulmonary tuberculosis," Infectious Diseases, vol. 33, no. 6, pp. 466-469, 2001.

[21] P. Kumar, A. Kaushik, D. T. Bell et al., "Mutation in an unannotated protein confers carbapenem resistance in Mycobacterium tuberculosis," Antimicrobial Agents and Chemotherapy, vol. 61, no. 3, Article ID e02234, 2017.

[22] O.-A. Abraham, V.-M. Rosa, S.-S. M. Antonio, G. Claudia, and M.-E. M. del Rosario, "Review of antibiotic and non-antibiotic properties of beta-lactam molecules," Anti-Inflammatory \& Anti-Allergy Agents in Medicinal Chemistry, vol. 15, no. 1, pp. 314, 2016.

[23] I. Dinçer, A. Ergin, and T. Kocagöz, "The vitro efficacy of $\beta$ lactam and $\beta$-lactamase inhibitors against multidrug resistant clinical strains of Mycobacterium tuberculosis," International Journal of Antimicrobial Agents, vol. 23, no. 4, pp. 408-411, 2004.

[24] M. Nakashima, J. Morita, and K. Aizawa, "Pharmakokinetics and safety of tebipenem pivoxil fidne granules, an oral carbapenem antibiotic, in healthy male volunteers," Japanese Journal of Chemotherapy, vol. 57, no. 1, pp. 90-94, 2009.

[25] C. M. Perry and T. Ibbotson, “Biapenem," Drugs, vol. 62, no. 15, pp. 2221-2234, 2002.

[26] A. D. Miller, A. M. Ball, P. B. Bookstaver, E. K. Dornblaser, and C. L. Bennett, "Epileptogenic potential of carbapenem agents: Mechanism of action, seizure rates, and clinical considerations," Pharmacotherapy, vol. 31, no. 4, pp. 408-423, 2011.

[27] G. G. Zhanel, R. Wiebe, L. Dilay et al., "Comparative review of the carbapenems," Drugs, vol. 67, no. 7, pp. 1027-1052, 2007.

[28] Y. Horita, S. Maeda, Y. Kazumi, and N. Doi, "In vitro susceptibility of Mycobacterium tuberculosis isolates to an oral carbapenem alone or in combination with $\beta$-Lactamase inhibitors," Antimicrobial Agents and Chemotherapy, vol. 58, no. 11, pp. 7010-7014, 2014.

[29] S. Hazra, H. Xu, and J. S. Blanchard, "Tebipenem, a new carbapenem antibiotic, is a slow substrate that inhibits the $\beta$ lactamase from Mycobacterium tuberculosis," Biochemistry, vol. 53, no. 22, pp. 3671-3678, 2014.

[30] R. Kobayashi, M. Konomi, K. Hasegawa, M. Morozumi, K. Sunakawa, and K. Ubukata, "In vitro activity of tebipenem, a new oral carbapenem antibiotic, against penicillin-nonsusceptible Streptococcus pneumoniae," Antimicrobial Agents and Chemotherapy, vol. 49, no. 3, pp. 889-894, 2005.

[31] C. Pitart, F. Marco, T. A. Keating, W. W. Nichols, and J. Vila, "Activity of ceftazidime-avibactam against fluoroquinoloneresistant Enterobacteriaceae and Pseudomonas aeruginosa," Antimicrobial Agents and Chemotherapy, vol. 59, no. 6, pp. 3059-3065, 2015.

[32] H. Xu, S. Hazra, and J. S. Blanchard, "NXL104 irreversibly inhibits the $\beta$-lactamase from Mycobacterium tuberculosis," Biochemistry, vol. 51, no. 22, pp. 4551-4557, 2012.

[33] J.-E. Hugonnet and J. S. Blanchard, "Irreversible inhibition of the Mycobacterium tuberculosis $\beta$-lactamase by clavulanate," Biochemistry, vol. 46, no. 43, pp. 11998-12004, 2007.

[34] A. R. White, C. Kaye, J. Poupard, R. Pypstra, G. Woodnutt, and B. Wynne, "Augmentin (amoxicillin/clavulanate) in the treatment of community-acquired respiratory tract infection: a review of the continuing development of an antimicrobial agent," Journal of Antimicrobial Chemotherapy, vol. 53, supplement 1, pp. i3-i20, 2004. 
[35] D. Zhang, Y. Wang, J. Lu, and Y. Pang, "In vitro activity of beta-lactams in combination with beta-lactamase inhibitors against multidrug-resistant Mycobacterium tuberculosis isolates," Antimicrob Agents Chemother, vol. 60, no. 1, pp. 393-399, 2015.

[36] L. W. Tremblay, F. Fan, and J. S. Blanchard, "Biochemical and structural characterization of Mycobacterium tuberculosis $\beta$ lactamase with the carbapenems ertapenem and doripenem," Biochemistry, vol. 49, no. 17, pp. 3766-3773, 2010.

[37] H. F. Chambers, D. Moreau, D. Yajko et al., "Can penicillins and other beta-lactam antibiotics be used to treat tuberculosis?" Antimicrobial Agents and Chemotherapy, vol. 39, no. 12, pp. 2620-2624, 1995. 


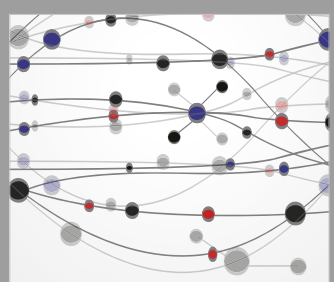

The Scientific World Journal
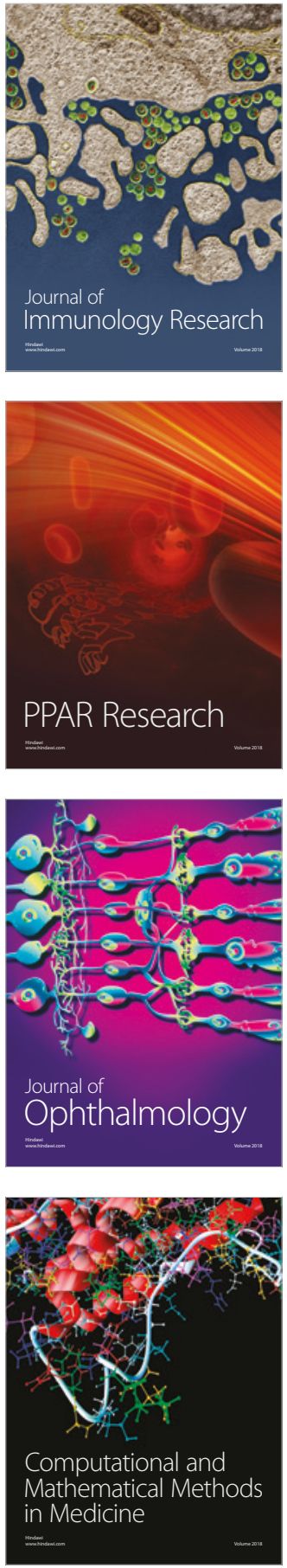

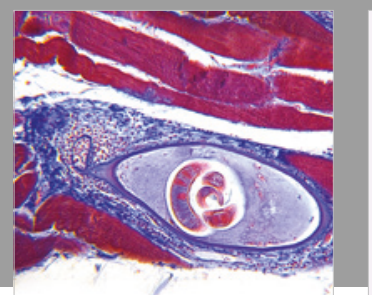

Gastroenterology Research and Practice

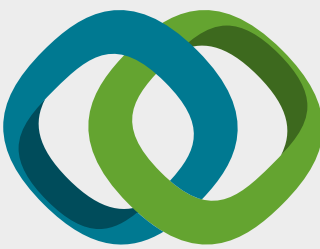

\section{Hindawi}

Submit your manuscripts at

www.hindawi.com
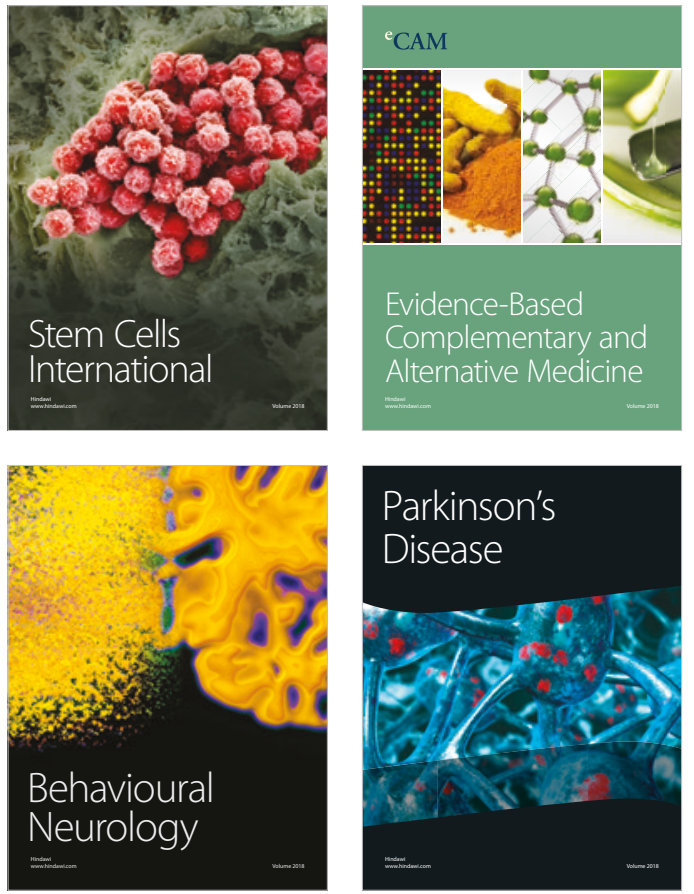

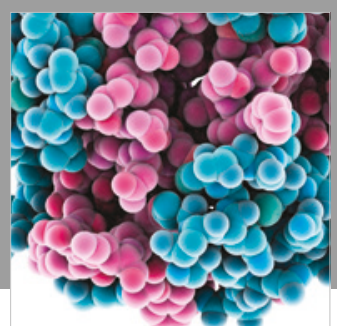

ournal of

Diabetes Research

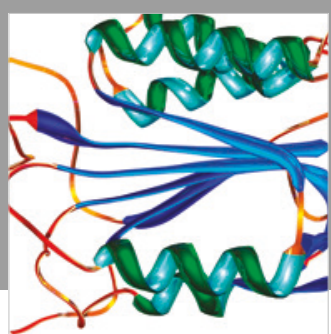

Disease Markers
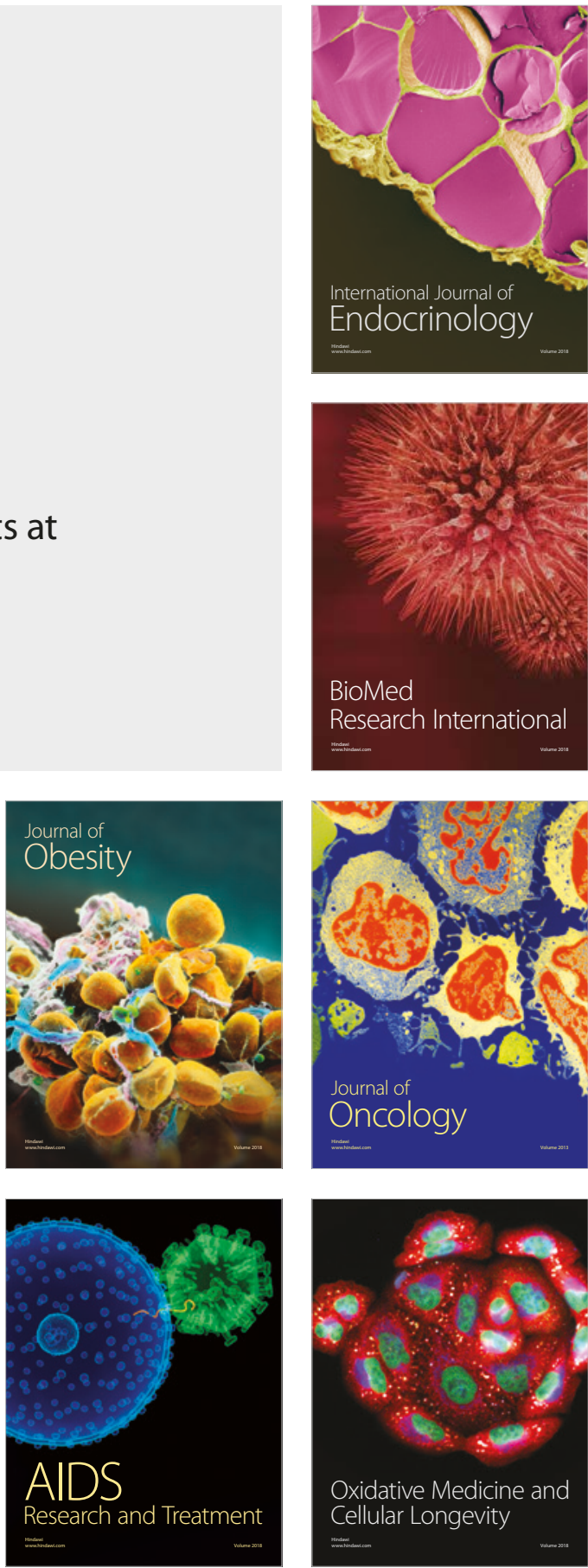